\title{
Genital HPVs in the aerodigestive tract: Etiologic association with a subset of oropharyngeal/tonsillar cancers and with recurrent respiratory papillomatosis ${ }^{1}$
}

\author{
Keerti V. Shah ${ }^{\mathrm{a}, *}$ and William H. Westra ${ }^{\mathrm{b}}$ \\ ${ }^{a}$ Department of Molecular Microbiology and Immunology, Bloomberg School of Public Health, Johns Hopkins \\ University, Baltimore, MD, USA \\ ${ }^{\mathrm{b}}$ Department of Pathology, School of Medicine, Johns Hopkins University, Baltimore, MD, USA
}

\section{Introduction}

The mucosal human papillomaviruses (HPVs) primarily infect the genital tract, but they also infect the mucosa lining the aerodigestive tract where they are etiologically linked to two clinical entities. The high-risk HPV type 16, which is responsible for about $50 \%$ of cervical cancers, is linked to a subset of oropharyngeal/ tonsillar cancers. Low-risk HPV types 6 and 11, which cause a large majority of genital warts, are also responsible for juvenile-onset as well as adult-onset recurrent respiratory papillomatosis (RRP).

\section{HPVs and Head and Neck Squamous Cell Carcinomas (HNSCC)}

Each year there are more than 550,000 newly diagnosed cases of HNSCC world wide, and these contribute to 300,000 annual cancer-related deaths [1]. Often considered a single and uniform tumor type, $\mathrm{HN}$ -

\footnotetext{
${ }^{1}$ This study was supported in part by grant number P50 CA96784 from the National Cancer Institute, NIH, Bethesda MD, USA.

* Corresponding author: $615 \mathrm{~N}$. Wolfe Street, Baltimore, MD 21205, USA. Tel.: +1 410955 3189; Fax: +1 443287 4563; E-mail: kvshah@jhsph.edu.
}

SCC in reality is a heterogeneous mix of cancers that differ in many ways including site of origin, causation, and behavior. Anatomically, HNSCCs originate from the mucosa lining the sinonasal tract, oral cavity, larynx and pharynx (inclusive of the nasopharynx, oropharynx and hypopharynx). The contribution of various risk factors is strongly influenced by social and ethnic factors. Cigarette smoking and alcohol consumption are by far the most widely recognized risk factors for HNSCC as a whole, but they are not the only ones. For example, oral cancers linked to betel quid chewing predominate in parts of India, and nasopharyngeal cancers associated with Epstein-Barr virus are common in China.

Infection with high-risk genital tract HPVs has long been established as the etiology of cancers of the female and male genital tracts, but its contribution to tumorigenesis of the head and neck has been an unsettled topic under intense investigation since the 1980s. Early studies reporting on the prevalence of HPV sequences in cancers and non-neoplastic tissues of the head and neck were marred by widely disparate results [2]. In one of the first studies to provide compelling evidence for the etiological role of HPV in HNSCC, Steenbergen et al. [3] observed the presence of integrated HPV-16 genome, HPV-16 transcripts and specific chromosomal alterations in both a primary oral squamous cell carci- 


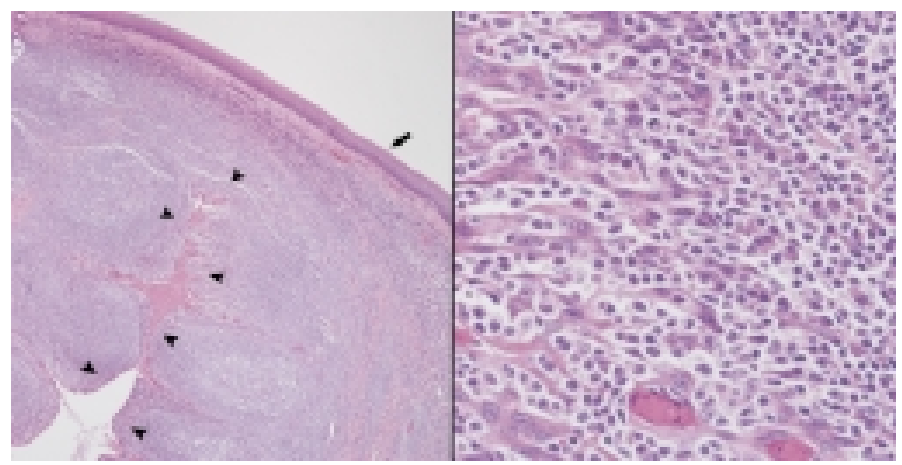

Fig. 1. Histology of the normal tonsil. The surface of the tonsil is lined by a layer of stratified squamous epithelium (left panel, arrows). Deep invaginations of the epithelium into the underlying lymphoid tissue are known as tonsillar crypts (left panel, arrowheads). The epithelium lining these crypts is disrupted by numerous permeating lymphocytes that separates the epithelium into cords of basaloid cells (right panel).

noma and in the cell line derived from the cancer. In the early 1990s, reports from Europe began to more clearly pinpoint the tonsil as an anatomic site targeted by HPV-16. Using an in-situ hybridization approach, Niedobitek et al. [4] frequently detected HPV-16 hybridization signals in HNSCC arising from the palatine tonsils. Using a PCR-based approach, Snidjers et al. [5,6] reported HPV-16 viral transcripts and evidence of HPV-16 integration in a high percentage of oropharyngeal carcinomas compared to HNSCCs arising in non-oropharyngeal sites.

As the role of HPV-16 in head and neck tumorigenesis becomes better understood, these HPV-16-related cancers are becoming increasingly recognized as a biologically distinct subgroup of HNSCCs with a characteristic pathological and clinical profile $[7,8]$. Compared to their non-HPV counterparts, HPV-16-related HNSCCs less frequently harbor inactivating mutations targeting the p53 and retinoblastoma $(\mathrm{Rb})$ tumor suppressor genes. Under the microscope, HPV-16-related HNSCCs consistently demonstrate a "basaloid" appearance in contrast to the conventional keratinizing morphology of most non-HPV-16-related HNSCCs. Patients with HPV-16-related HNSCCs are less likely to use alcohol or tobacco, and they are more likely to experience favorable outcomes. As first suggested by Niedobitek and others [4], another distinctive feature of HPV-16-related HNSCC is its strong tendency to localize to the oropharynx - an anatomic site that includes the base of the tongue, lingual and palatine tonsils, soft palate and uvula. Within the oropharynx, HPV-16 appears to target the specialized reticulated epithelium that lines the tonsillar crypts (Fig. 1).

Several recent reviews have discussed different aspects of the role of HPVs in tumors of the aerodigestive tract [8-10]. This review will summarize the charac- teristics of HNSCC etiologically linked to HPV infections.

\subsection{Virologic characteristics}

Kreimer et al. [9] systematically reviewed 60 PCRbased studies to describe the prevalence, viral genotypes, and anatomic distribution of HPVs in over 5000 HNSCCs. In the combined results of the studies, the prevalence of HPV DNA in squamous cell carcinomas of the oropharynx $(36 \%)$ was significantly higher than that in squamous cell carcinomas of the oral cavity (24\%) and larynx (24\%). For all three groups, HPV16 was by far the most common HPV type detected in the HPV-positive carcinomas. The predominance of HPV-16 type in HNSCC is a common finding in the PCR-based studies.

These PCR-based studies reported the presence of HPV-16 in HNSCCs across various anatomic sites, but were unable to describe the distribution of the viral genome in tumor tissues. Unlike PCR-based detection methods, current in situ hybridization methods permit direct visualization of HPV-16 in tumor cells. Moreover, the in situ methods are highly sensitive and are able to detect a single HPV DNA copy per cell and they are applicable to formalin-fixed paraffinembedded clinical samples. Punctate staining in the nucleus of the tumor cell is suggestive of an integrated state of the viral genome. Begum et al. [11] performed in situ hybridization for HPV-16 DNA on a tissue array assembled from HNSCCs representing all anatomic subsites of the head and neck. They detected HPV-16 in $82 \%$ of the oropharyngeal cancers but in only one of 131 cancers $(0.8 \%)$ arising from non-oropharyngeal sites. All 115 cancers from the oral cavity and the larynx were HPV-16 negative. The HPV prevalence data comparing 
Table 1

HPV prevalence in tumor biopsies from different HNSCC sites by PCR and by in situ hybridization

\begin{tabular}{|c|c|c|c|c|c|c|c|}
\hline \multirow[t]{2}{*}{ Study } & \multirow[t]{2}{*}{ Assay } & \multicolumn{2}{|c|}{ Oropharynx } & \multicolumn{2}{|c|}{ Oral Cavity } & \multicolumn{2}{|c|}{ Larynx } \\
\hline & & No. & (\% prev) & No. & (\% prev) & No. & (\% prev) \\
\hline Kreimer et al. [9] & PCR for all HPVs & 969 & $(35.6)$ & 2642 & $(23.5)$ & 1435 & $(24.0)$ \\
\hline Kreimer et al. [9] & PCR for HPV-16 & 969 & $(30.9)$ & 2642 & $(16.0)$ & 1535 & $(16.6)$ \\
\hline Begum et al. [11] & ISH for HPV-16 in tumor cell nuclei & 45 & $(82)$ & 61 & $(0)$ & 54 & $(0)$ \\
\hline
\end{tabular}
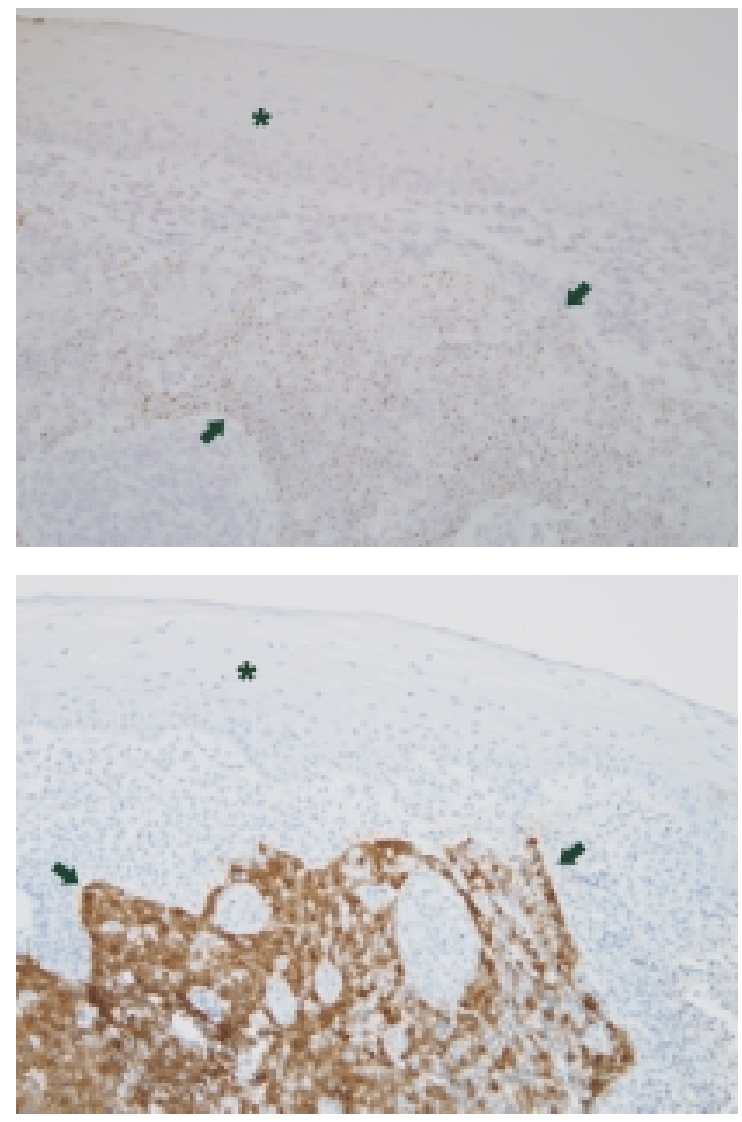

Fig. 2. HPV16 in-situ hybridization and p16 immunohistochemistry of a tonsillar carcinoma. Distinct dot-like hybridization signals are localized to the tumor nuclei involving a tonsillar crypt (outlined by arrows), but they are not present in the overlying surface epithelium (asterisk) (A, HPV in-situ hybridization). The pattern of high p16 expression reflects the distribution of HPV virus (B, p16 immunohistochemistry).

PCR-based detection and in situ hybridization detection across three anatomic sites [11] are shown in Table 1. They show that although cancers at all three HNSCC sites are HPV-positive by PCR, only the oropharyngeal cancers demonstrate the presence of HPV as visualized by hybridization signals within the tumor nuclei (Fig. 2A). The same conclusion was reached in a study of paraffin sections of metastatic lymph nodes from 68 HNSCC patients by in situ hybridization [12]. HPV-16 DNA was found in the tumor nuclei of 22 of 31 patients $(71 \%)$ whose primary tumor was in the oropharynx, but in none of 37 patients with primary tumors at other sites (21 in oral cavity, 11 in larynx and 5 in hypopharynx). In situ hybridization was performed only with HPV-16 probe; thus these studies likely underestimate the true prevalence of HPV-associated cancers by missing the few oropharyngeal cancers associated with some other HPV type. In confirmation of the in situ hybridization studies, high copy number of HPV DNA and the presence of HPV E6 and E7 transcripts strongly correlate with tumor origin from the oropharynx [6,13-16]. Gillison et al. [13] tested HPV PCR-positive HNSCC biopsies by in situ hybridization and/or Southern hybridization of tissue DNAs. They found that PCR-positive oropharyngeal cancers were much more likely to be positive by either or both of these assays (17 of $24(71 \%)$ ) than PCR-positive biopsies from non-oropharyngeal HNSCC sites (one of 13 $(8 \%))$.

With respect to the physical state of HPV-16 DNA in HPV-positive oropharyngeal/tonsillar cancers, evidence has been presented for the viral genome being predominantly episomal [14] as well as for its being integrated $[6,8,15]$.

In summary, the majority of squamous cell carcinomas arising from the oropharynx have a transcriptionally active HPV genome localized to the tumor cells, suggesting that they are a result of a clonal expansion of HPV-transformed cells. Tumors with these characteristics appear to be virtually absent from other HNSCC anatomic sites such as the oral cavity and the larynx. Although squamous cell carcinomas of the larynx can arise from malignant transformation of recurrent respiratory papillomas caused by the non-oncogenic HPV types 6 and 11, this is an exceedingly rare phenomenon [17]. HNSCCs which have small copy numbers of HPV DNA by quantitative PCR assays, or HNSCCs that are HPV-negative by in situ hybridization, are very likely not etiologically linked to HPV infection.

\subsection{Pathogenesis}

\subsubsection{Pathological aspects}

The HPV-associated HNSCCs have a characteristic appearance under the microscope that is distinct 


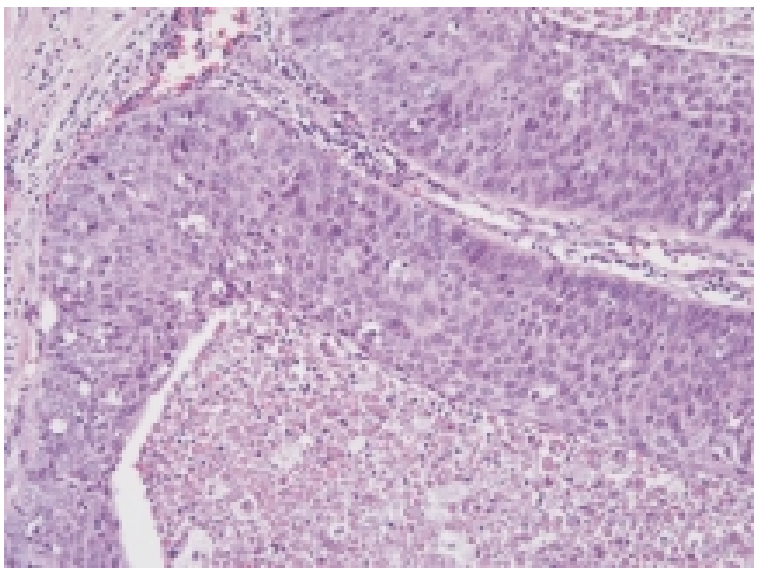

Fig. 3. Characteristic basaloid appearance of an HPV16-related oropharyngeal carcinoma. The carcinoma grows as expanding cellular lobules with central cystic necrosis. The tumor cells are non-keratinizing and have a high nuclear to cytoplasmic (N:C) ratio.

from their non-HPV-related counterparts. In contrast to the keratinizing morphology of conventional HNSCC, these HPV-related tumors tend to be seen as expanding lobules of poorly differentiated and non-keratinizing squamous cells, imparting a "basaloid" appearance [15] (Fig. 3). Of note, this same basaloid morphology has also been observed in the HPV-related squamous carcinomas of the vulva and penis [18].

The consistent localization of HPV-related HNSCC to the oropharynx may reflect viral targeting of the epithelium lining the tonsillar crypts [16]. This specialized lymphoepithelium has a distinctly "basaloid" appearance, is involved in the capture and processing of antigens, and serves as a replication site for important pathogenic viruses (e.g., Epstein-Barr virus, adenoviruses, and herpes simplex virus) and perhaps may serve as a reservoir for HPV following exposure [19]. In their description of the HPV-16 distribution in oropharyngeal carcinomas as visualized by in situ hybridization, Begum et al. [11] found that all of the $37 \mathrm{HPV}$-positive oropharyngeal carcinomas arose either from the lingual $(n=17)$ or palatine $(n=20)$ tonsils, whereas four of the eight HPV-negative oropharyngeal cancers arose from the soft palate, a site unassociated with crypt epithelium. In those HPV-positive cases where all histologic stages of tumor progression were present from epithelial dysplasia to invasive carcinoma, the premalignant changes localized to the tonsillar crypts and were HPV-16 positive. These observations suggest that HPV-16 targets the tonsillar crypt epithelium for the initiation and maintenance of the malignant phenotype.
The propensity of HPV-16 to initiate tumorigenesis from deep within the tonsillar crypts may help account for certain well recognized but poorly understood clinical characteristics of tonsillar carcinoma. First, premalignant changes of the tonsils are rarely clinically evident. This inability to visualize tonsillar dysplasia diminishes the opportunity to remove the tonsils during the early stages of tumor development. Second, tonsillar carcinomas are prone to metastatic spread to regional lymph nodes even when the surface of the tonsils appears visibly unremarkable [20]. Indeed, a significant number of metastatic carcinomas from occult primary tumors are HPV-16 positive (Westra, unpublished results), likely reflecting origin from the deep recesses of the tonsillar crypts. It is not known if this location of HPV infection to the tonsillar crypts will adversely affect the ability to detect HPV as part of screening protocols for early cancer detection. Of note, HPV is readily recovered from exfoliated cervical cells, but it is not consistently recovered in oral rinses obtained from patients with HPV-positive HNSCCs [21].

\subsubsection{Molecular aspects}

Tumorigenesis of the head and neck almost always involves inactivation of the $\mathrm{p} 53$ and $\mathrm{Rb}$ pathways - two pathways that are intimately involved in regulation of the cell cycle [22]. In non-HPV-related HNSCCs, disruption of the p53 pathways occurs via genetic alterations targeting the p53 gene; and disruption of the $\mathrm{Rb}$ pathway predominantly occurs via genetic and/or epigenetic silencing of the p16(INK4A) tumor suppressor gene. In HPV-related HNSCCs, genetic alterations targeting p53 and p16(INK4A) are much less common. Instead, the viral oncoproteins E6 and E7 are expressed and they physically bind and functionally inactivate the p53 and pRb proteins, respectively. Andl et al. [7] were among the first to note the disparity between $\mathrm{Rb}$ protein expression and $\mathrm{Rb}$ mutational status as a function of HPV infection. Compared to other HNSCCs, Rb protein expression was disproportionately reduced in the tonsillar carcinoma, even though these carcinomas lacked $\mathrm{Rb}$ mutations. HPV DNA was identified in 11 of 12 tonsillar carcinomas with reduced $\mathrm{Rb}$ expression, but in none of nine tonsillar carcinomas with normal levels of $\mathrm{Rb}$ protein. A growing number of studies have also consistently shown that HPV-associated oropharyngeal carcinomas are much less likely to harbor p53 mutations than oropharyngeal carcinomas that are not HPV related [13].

In HPV-positive cervical carcinomas, transcription of the viral oncoprotein E7 physically binds and func- 
tionally inactivates the Retinoblastoma $(\mathrm{Rb})$ gene product, causing a perturbation of other key components of the $\mathrm{Rb}$ pathway. As one example, functional inactivation of Rb by E7 is known to induce an upregulation of p16 expression [23], reaching levels that can be readily detected by routine immunohistochemistry (IHC) [24]. In effect, immunohistochemical detection of high p16 expression serves as a surrogate marker of infection with high-risk HPVs and, in turn, a marker for neoplastic transformation of the cervix [25]. Immunohistochemical detection of high p16 expression is also an effective ancillary tool for identifying HPV-associated HNSCC. Presence of p16 by IHC correlates very well with the presence of HPV genome by in situ hybridization [26] (Fig. 2B). Testing of paraffin-embedded tissue sections for both HPV-16 in situ hybridization and p16 IHC is an effective way to screen for HPV-associated HNSCC; positive results in both assays would be expected for most of the HPV-associated HNSCC, and positive results only for p16 IHC may indicate HNSCC associated with HPVs other than HPV-16.

Other differences between HPV-associated and HPV-unassociated HNSCC have been described. Compared with their non-HPV-related counterparts, HPVrelated HNSCC are less likely to be associated with inactivation of the tumor suppressor RASSFIA [27], less likely to be associated with inactivation of 14-3-3 sigma, a regulator of senescence in keratinocytes [28]; and less likely to exhibit gross chromosomal changes [29].

\subsection{Serologic correlates}

Mork et al. [30] conducted a case-control study nested in a large Nordic cohort to examine if the presence of antibodies to HPV-16 and some other HPV types in pre-diagnostic sera (a measure of previous HPV infection) was a risk factor for subsequent HNSCC. They found that antibodies reactive with HPV-16 VLPs in pre-diagnostic sera (on average 9.4 years before diagnosis) conferred a high14-fold risk for oropharyngeal cancers and a lower risk for HNSCC at other anatomic sites. They also examined 160 archived HNSCC tumors for HPV DNA and found HPV-16 DNA in 15 tumors. Detection of HPV-16 DNA in the tumors correlated with pre-diagnostic seropositivity to HPV-16 VLPs.

Antibodies to HPV oncoproteins E6 and E7 are markers of HPV-associated invasive cancer. In a casecontrol study of women with invasive cervical cancer, Sun et al. [31] found that $63 \%$ of cases and $10 \%$ of controls had HPV-16 E6 and/or E7 antibodies, and that
$21 \%$ of cases and $0.5 \%$ of controls had antibodies for both HPV-16 E6 and E7. Similar patterns of E6/E7 antibody prevalence are seen with HNSCC. Zumbach et al. [32] reported the presence of HPV-16 E6/E7 antibodies in 7 of $19(37 \%)$ HNSCC patients who had HPV-16 DNA in their tumors and lower prevalences in other groups. In the large international multicenter study [21], the seroprevalence of HPV-16 E6/E7 antibodies was high $(65.4 \%)$ in patients with HPV DNApositive oropharyngeal cancers, and low (3.2-4.2\%) in patients with HPV DNA-negative oropharyngeal cancers, in patients with oral cancers and in individuals without cancer.

In summary, serologic studies have established that HPV-16 infection precedes the development of HPV16 DNA-positive HNSCC and that patients with HPV16-positive oropharyngeal cancer develop antibodies which are markers of HPV-caused cancers.

\subsection{Clinical features}

Several studies have shown that patients with HPVpositive HNSCCs have a better prognosis than patients with HPV-negative HNSCCs [13]. Mellin et al. [33] compared the clinical outcomes of patients with HPV16-positive $(n=23)$ and HPV-negative $(n=29)$ tonsillar carcinomas, and found that the risk of cancer-related death was reduced by one-half in the HPV-positive group. In the group of patients with oropharyngeal carcinoma reported by Gillison et al. [13], HPV-positivity was associated with fewer cancer related deaths (3 of 24 HPV-positive cases vs. 10 of 26 HPV-negative cases) and significantly improved disease specific survival. Of the 99 cases of oropharyngeal carcinomas reported by Lindel et al. [34], the 14 HPV-positive cases were associated with a lower recurrence rate and longer patient survival. Li et al. [35] studied 67 patients with tonsillar cancer, 31 of whom had HPV-positive tumors. They found that patients with HPV-positive tumors were 3 times less likely to have recurrence and 5 times less likely to die of the disease than patients with HPV-negative tumors. Weinberger et al. [36] divided 77 patients with oropharyngeal cancers into three groups based on the HPV-16 and p16 status of their tumors. Patients who had HPV-16-positive tumors with high p16 expression (18 cases, group 3 ) had a better prognosis (five year survival 79\%) than patients with HPV-16-negative tumors with low p16 expression (30 cases, group 1, five year survival 20\%) or patients with HPV-16-positive tumors with low p16 expression (29 cases, group 2, five year survival, 18\%). The HPV-16 viral load was ten-fold 
Table 2

Characteristics of HPV-associated HNSCC

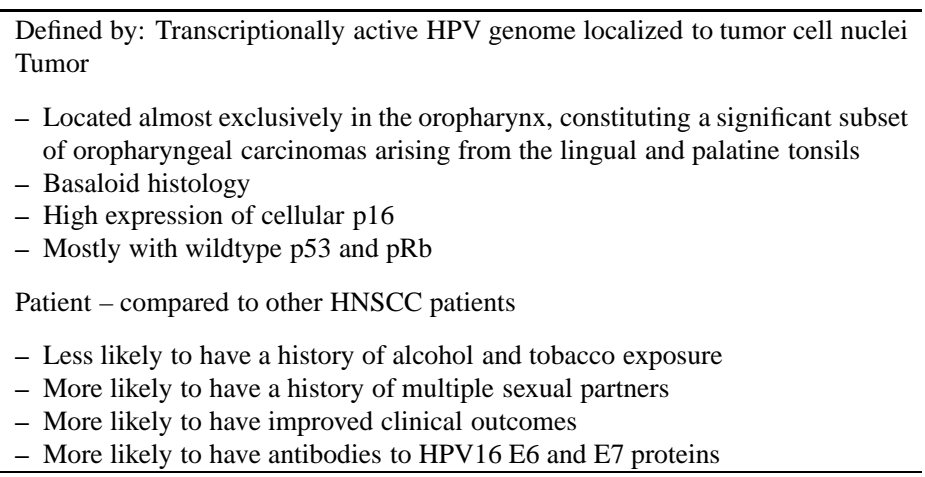

higher in group 3 tumors (median, 46 copies per cell) as compared to group 2 tumors (median 3.6 copies per cell). It is possible that the group 3 patients in this study had tumors etiologically linked to HPV-16 and that patients in group 2, with low copy numbers of HPV-16, had tumors not etiologically linked to HPV-16.

The biology underlying the clinical observation of improved prognosis for patients with HPV-associated HNSCC remains to be clarified. Improved outcomes associated with HPV infection is thought to be related to any combination of multiple factors including immune modulation, increased tumor sensitivity to chemoradiation, and a less complex profile of genetic alterations.

\subsection{Epidemiologic findings}

Patients with HPV-associated oropharyngeal carcinomas are less likely to have a history of exposure to alcohol and tobacco than patients with non-HPVassociated HNSCCs [8].

Risk factors for oral HPV infections are related to sexual behavior and include orogenital contact, multiple sexual partners and HIV infection [37]. In healthy individuals, the prevalence of HPV DNA sequences in tonsillar epithelium is quite low and estimated to be $1 \%$ by Kreimer et al. [38] and $0.64 \%$ by Hansson et al. [39]. HPV is more prevalent in tonsillar epithelium and in oral rinse specimens in HIV-infected individuals compared to the general population [38]. Further, HPV-associated carcinomas are more likely to occur in HIV-infected individuals than in the general population [40].

\subsection{Magnitude of the problem}

In his estimates of the global burden of infectionrelated cancers in 2002, Parkin [41] used a combina- tion of HPV PCR and E6/E7 serology data from cases and controls in the international study of HNSCCs [21] to suggest that, worldwide, $3 \%$ of oral cancers $(8,200$ cases) and $12 \%$ of oropharyngeal cancers $(6,300$ cases) were attributable to HPV infections. By the criteria described earlier in this review (transcriptionally active HPV genome or HPV genome localized to tumor cells), at least one half of all oropharyngeal cancers and practically no oral cavity cancers in the USA and Europe are HPV-associated. If the contribution of HPVs to oropharyngeal cancers is similar elsewhere in the world, about 25,000 of the 51,200 oropharynx cases worldwide might be HPV-related. However, the contribution of HPV infections to these cancers may be geographically variable. Li et al. [35] have reported that 31 of 67 (46\%) tonsillar cancers from Australia, but none of 16 tonsillar cancers from China, were HPV-positive by PCR. Also, the incidence of tonsillar cancers has been increasing over the past few decades [42] and it has been suggested that this may be, in part, because of the increasing contribution of HPVs to tonsillar cancer over time [43]. Thus, a crude estimate of HPVassociated cancers worldwide may be in the range of 15,000-25,000 per year.

\subsection{Prevention and control}

Both of the recently introduced Merck and Glaxo Smith Kline (GSK) HPV-based prophylactic vaccines against cervical cancer include HPV-16 and HPV-18 VLPs. In clinical trials in young women, both vaccines have been shown to be nearly $100 \%$ effective in preventing incident HPV-16 and HPV-18 infections and cervical intraepithelial neoplasia (CIN) associated with these types. Trials of the effectiveness of the vaccines in men are in progress. Because the vaccines prevent incident infections, it is very likely that wide-spread 
Table 3

Comparison of HPV-associated oropharyngeal squamous cell carcinoma with squamous cell carcinoma of the cervix

\begin{tabular}{lll}
\hline & $\begin{array}{l}\text { HPV-associated squamous cell } \\
\text { carcinoma of the oropharynx }\end{array}$ & $\begin{array}{l}\text { HPV-associated squamous cell } \\
\text { carcinoma of cervix }\end{array}$ \\
\hline Attributed to HPVs & $\begin{array}{l}\text { At least 50\% of } \\
\text { oropharyngeal cancers }\end{array}$ & $100 \%$ \\
$\begin{array}{l}\text { Attributed to HPV-16 } \\
\text { Site of precursor lesion } \\
\text { Gender }\end{array}$ & $\begin{array}{l}\text { tonsillar crypt } \\
\text { male predominance }\end{array}$ & $\begin{array}{l}50 \% \text { of HPV-positive } \\
\text { transformation zone } \\
\text { Females }\end{array}$ \\
\hline
\end{tabular}

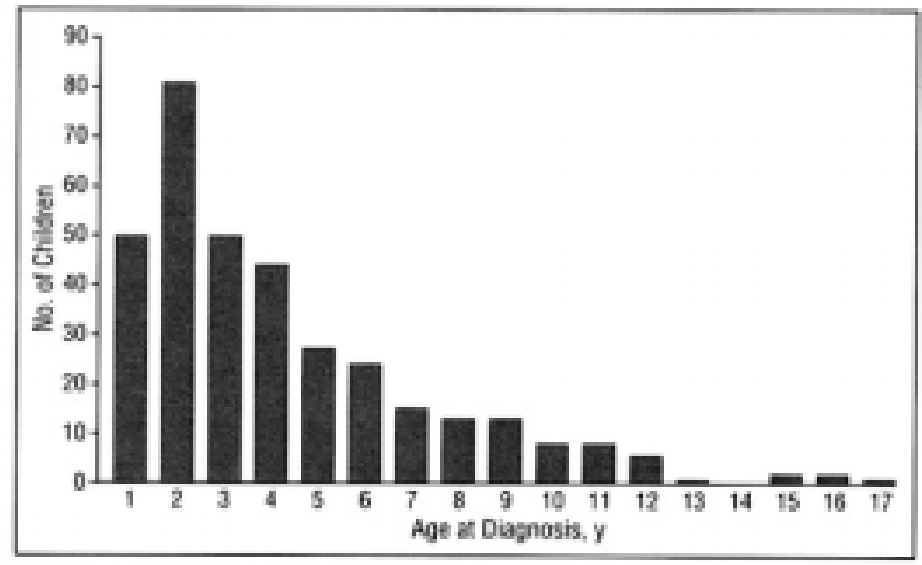

Distribution of age at diagnosis for children enrolied in the recurrent respiratory papillomatosis registry.

Fig. 4. Distribution of age at diagnosis for children enrolled in the recurrent respiratory papillomavirus registry (from [54]).

utilization of these vaccines will reduce the burden of HPV-associated HNSCC.

A number of HPV-16 E6- and E7-based therapeutic vaccines have shown promise in animal models where they protect mice against challenge with HPV16-transformed tumor cells [44]. Some of these vaccines are in trials in patients with HPV-16-associated oropharyngeal cancers.

\subsection{Summary}

The characteristics of HPV-associated HNSCC are summarized in Table 2. A comparison of some aspects of HPV-associated squamous cell carcinomas of the oropharynx and the cervix is shown in Table 3.

\section{HPVs and Recurrent Respiratory Papillomatosis (RRP)}

Genital HPVs are also etiologically linked to the benign condition, recurrent respiratory papillomatosis, in the aerodigestive tract. RRP may have onset in childhood (juvenile-onset RRP (JORRP)) with most of the cases occurring in the first 4 years of life (Fig. 4), or in adult life (adult-onset-RRP (AORRP)) with a small peak at 21-30 years.

\subsection{Virologic characteristics}

The non-oncogenic HPV types 6 and 11 that commonly infect the genital tract and are responsible for a large majority of genital warts (condylomas) also account for almost all cases of JORRP and AORRP.

\subsection{Pathogenesis}

For JORRP, the transmission of HPV-6 or HPV-11 most likely occurs intra-partum from mother to child when the fetus is passing through an infected birth canal. This mode of transmission is similar to that of neonatal transmission of herpes simplex virus 2 . The manner of transmission in AORRP is not known but it is unlikely that the infection is acquired at birth. 
In a child with JORRP, latent HPV infection is apparently widespread throughout the respiratory tract [45]. Papillomas are most frequently found on the vocal cords in the larynx, but may occur at any other site in the respiratory tract. In the genital tract, infection with HPV-6 is more frequent than with HPV-11, but HPV-11 is responsible for a majority of JORRP cases, suggesting that HPV-11 is transmitted more readily than HPV6 from mother to child. HPV-11 is associated with a more severe clinical course of JORRP [46], as well as with a greater likelihood of malignant transformation of the papilloma [47].

\subsection{Clinical features}

The course of the disease is highly variable. Although the papillomas are benign and malignant conversion is rare, the recurrent nature of the disease, and the need for repeated surgical interventions to keep the airways open, often impose an enormous burden on the patients and their families (Fig. 5). The number of surgeries required is highest in children with an early onset of disease (Fig. 6), and in severe cases, may exceed 100 [48]. Extension of the disease to the trachea and lungs is a serious complication and it sometimes results from the seeding of infected cells at these sites by the repeated surgical procedures.

\subsection{Epidemiologic findings}

Maternal condyloma during pregnancy is the overwhelming risk factor for JORRP. About $50 \%$ of the mothers of JORRP cases give a history of genital warts during pregnancy [49]. In a population-based study in Denmark [50], maternal condyloma during pregnancy was estimated to increase the risk of JORRP in the child by more than 200 fold. However, the risk of JORRP in a child born to a mother who had condyloma in pregnancy was not large and was estimated to be less than $1 \%$, and a majority of JORRP cases were born to mothers who did not have a history of condyloma during pregnancy.

\subsection{Magnitude of the problem}

The estimates of the number of JORRP cases in the USA, based on extrapolations from questionnaire data and from a small population-based study are imprecise and have varied from a low of 80 to a high of 2340 [51]. The number of reported cases of JORRP in one survey, without extrapolations, was 840 for the year 1976 [52].
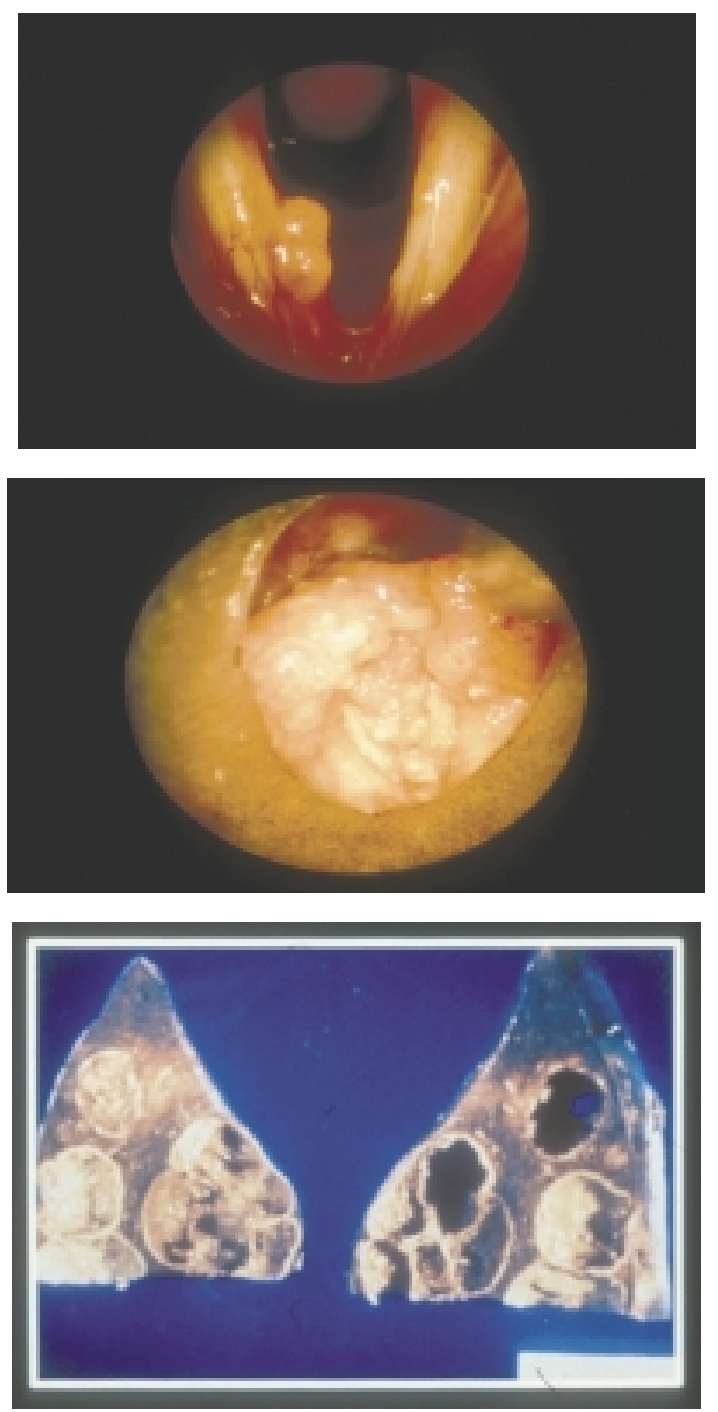

Fig. 5. Clinical presentations of RRP: (a) papilloma on the vocal cord; (b) papilloma obstructing the airway; and (c) papilloma spreading into lungs.

\subsection{Prevention and control}

Cesarean delivery of the at-risk child might be helpful in preventing JORRP, but it has not been possible to directly evaluate this possibility, because the disease is rare, and the frequency of JORRP even in the high-risk group is low. Indirect evidence for the effectiveness of cesarean delivery has been sought by comparing the cesarean delivery rate in mothers of JORRP cases with national rates [51]. While the data were not consistent across different studies, they suggest that cesarean delivery may afford some protection. 


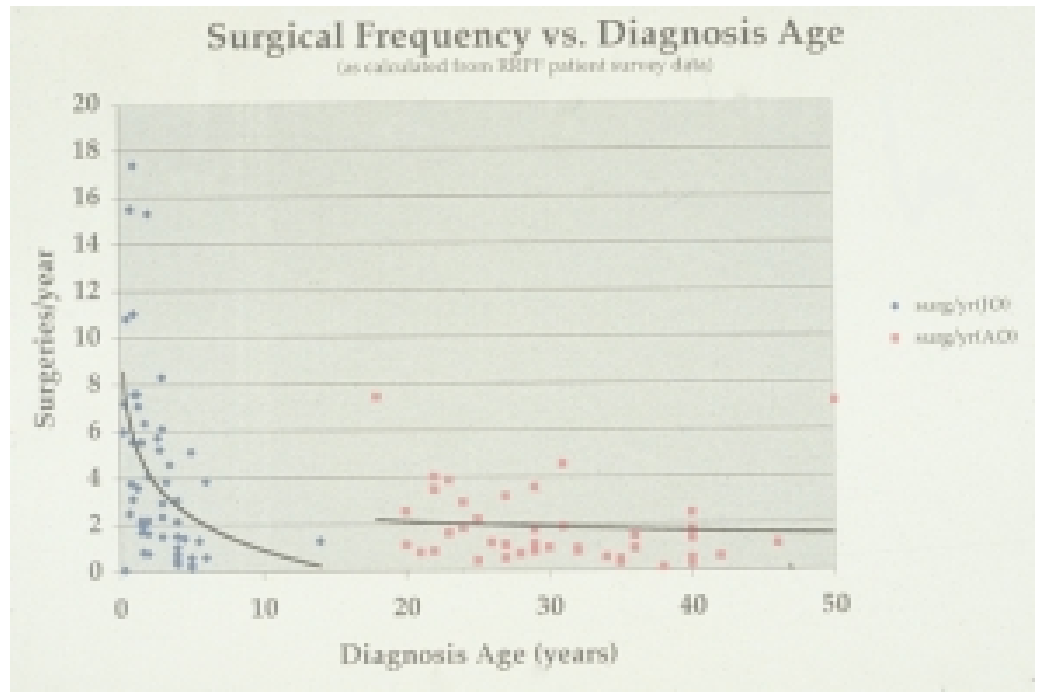

Fig. 6. Number of annual surgeries by age of patient in the Recurrent Respiratory Papillomavirus Foundation Registry (courtesy of Bill Stern).

Prophylactic HPV VLP-based vaccines which include HPV-6 and HPV-11 VLPs, such as Merck's Gardasil ${ }^{\circledR}$, are anticipated to completely change the landscape for prevention of both JORRP and AORRP. In clinical trials, Gardasil ${ }^{\circledR}$ has been shown to be very effective in protecting young previously uninfected women against incident HPV-6 and HPV-11 infections and against genital warts associated with these two HPV types [53]. Women immunized with Gardasil ${ }^{\circledR}$ are likely to be fully protected against having a child with JORRP. Currently, the Merck Gardasil ${ }^{\circledR}$ vaccine approved by the US FDA is recommended for pre-teen and young women. But older women, pregnant women who have condyloma during pregnancy and even infants born to women who had condyloma during pregnancy could conceivably benefit from the vaccine [51]. Surveillance for JORRP after the introduction of the vaccine will be necessary to evaluate the effectiveness of the vaccine in preventing JORRP.

\subsection{Summary}

JORRP and AORRP are significant public health problems which at present are difficult to prevent or treat. The HPV VLP-based prophylactic vaccines which include HPV-6 and HPV-11 VLPs have great promise for the reduction of the burden of these diseases.

\section{References}

[1] D.M. Parkin, F. Bray, J. Ferlay and P. Pisani, Global cancer statistics, 2002, CA Cancer J Clin 55(2) (2005), 74-108.
[2] S. Franceschi, N. Muñoz, F.X. Bosch, P.J.F. Snijders and J.M.M. Walboomers, Human papillomavirus and cancers of the upper aerodigestive tract: A review of epidemiological and experimental evidence, Cancer Epidemiol Biomarkers Prev 5 (1996), 567-575.

[3] R.D.M. Steenbergen, A.J.A. Hermsen, J.M.M. Walboomers, H. Joenje, F. Arwert, C.J.L.M. Meijer et al., Integrated human papillomavirus type 16 and loss of heterozygosity at $11 \mathrm{q} 22$ and $18 \mathrm{q} 21$ in an oral carcinoma and its derivative cell line, Cancer Res 55 (1995), 5465-5471.

[4] G. Niedobitek, S. Pitteroff, H. Herbst, P. Shepherd, T. Finn, I. Anagnostopoulos et al., Detection of human papillomavirus type 16 DNA in carcinomas of the palatine tonsil, J Clin Pathol 43 (1990), 918-921.

[5] P.J.F. Snijders, F.V. Cromme, A. van den Brule, H.F.J. Schrijnemakers, G.B. Snow and C.J.L.M. Meijer, Prevalence and expression of human papillomavirus in tonsillar carcinomas, indicating a possible viral etiology, Int J Cancer 51 (1992), $845-850$.

[6] P.J.F. Snijders, C.J.L.M. Meijer, A. van den Brule, H.F.J. Schrijnemakers, G.B. Snow and J.M.M. Walboomers, Human papillomavirus (HPV) type 16 and 33 E6/E7 region transcripts in tonsillar carcinomas can originate from integrated and episomal HPV DNA, J Gen Virol 73 (1992), 2059-2066.

[7] T. Andl, T. Kahn, A. Pfuhl, T. Nicola, R. Erber, C. Conradt et al., Etiological involvement of oncogenic human papillomavirus in tonsillar squamous cell carcinomas lacking retinoblastoma cell cycle control, Cancer Res 58 (1998), 5-13.

[8] M.L. Gillison, Human papillomavirus-associated head and neck cancer is a distinct epidemiologic, clinical, and molecular entity, Semin Oncol 31(6) (2004), 744-754.

[9] A.R. Kreimer, G.M. Clifford, P. Boyle and S. Franceschi, Human papillomavirus types in head and neck squamous cell carcinomas worldwide: a systematic review, Cancer Epidemiol Biomarkers Prev 14(2) (2005), 467-475.

[10] P.J.F. Snijders, A. van den Brule, C. Meijer and J. Walboomers, HPV and cancer of the aerodigestive tract, Papillomavirus Rep 6 (1995), 157-162. 
[11] S. Begum, D. Cao, M. Gillison, M. Zahurak and W.H. Westra, Tissue distribution of human papillomavirus 16 DNA integration in patients with tonsillar carcinoma, Clin Cancer Res 11(16) (2005), 5694-5699.

[12] S. Begum, M.L. Gillison, M.A. Ansari-Lari, K. Shah and W.H. Westra, Detection of human papillomavirus in cervical lymph nodes: a highly effective strategy for localizing site of tumor origin, Clin Cancer Res 9(17) (2003), 6469-6475.

[13] M.L. Gillison, W.M. Koch, R.B. Capone, M.D. Spafford, W.H. Westra, L. Wu et al., Evidence for a causal association between human papillomavirus and a subset of head and neck cancers, JNCI 92 (2000), 709-720.

[14] H. Mellin, L. Dahlgren, E. Munck-Wikland, J. Lindholm, H. Rabbani, M. Kalantari et al., Human papillomavirus type 16 is episomal and a high viral load may be correlated to better prognosis in tonsillar cancer, Int J Cancer 102(2) (2002), 152158.

[15] S. Wilczynski, B. Lin, Y. Xie and B. Paz, Detection of human papillomavirus DNA and oncoprotein overexpression are associated with distinct morphological patterns of tonsillar squamous cell carcinoma, Am J Pathol 152 (1998), 145-156.

[16] I. Paz, N. Cook, T. Odom-Maryon et al., Human papillomavirus (HPV) in head and neck cancer. An association of HPV 16 with squamous cell carcinoma of Waldeyer's tonsillar ring, Cancer 79 (1997), 595-604.

[17] C.G. Hobbs and M.A. Birchall, Human papillomavirus infection in the etiology of laryngeal carcinoma, Curr Opin Otolaryngol Head Neck Surg 12(2) (2004), 88-92.

[18] M. Sideri, R.W. Jones, E.J. Wilkinson, M. Preti, D.S. Heller, J. Scurry et al., Squamous vulvar intraepithelial neoplasia: 2004 modified terminology, ISSVD Vulvar Oncology Subcommittee, J Reprod Med 50(11) (2005), 807-810.

[19] I. Brook, The clinical microbiology of Waldeyer's ring, Otolaryngol Clin North Am 20(2) (1987), 259-272.

[20] I. Haas, T.K. Hoffmann, R. Engers and U. Ganzer, Diagnostic strategies in cervical carcinoma of an unknown primary (CUP), Eur Arch Otorhinolaryngol 259(6) (2002), 325-333.

[21] R. Herrero, X. Castellsague, M. Pawlita, J. Lissowska, F. Kee, P. Balaram et al., Human papillomavirus and oral cancer: the International Agency for Research on Cancer multicenter study, J Natl Cancer Inst 95(23) (2003), 1772-1783.

[22] A. Forastiere, W. Koch, A. Trotti and D. Sidransky, Head and neck cancer, $N$ Engl J Med 345(26) (2001), 1890-1900.

[23] M. Serrano, G.J. Hannon and D. Beach, A new regulatory motif in cell-cycle control causing specific inhibition of cyclin D/CDK4, Nature 366(6456) (1993), 704-707.

[24] J.P. Klussmann, E. Gultekin, S.J. Weissenborn, U. Wieland, V. Dries, H.P. Dienes et al., Expression of p16 protein identifies a distinct entity of tonsillar carcinomas associated with human papillomavirus, Am J Pathol 162(3) (2003), 747-753.

[25] R. Klaes, T. Friedrich, D. Spitkovsky, R. Ridder, W. Rudy, U. Petry et al., Overexpression of p16(INK4A) as a specific marker for dysplastic and neoplastic epithelial cells of the cervix uteri, Int J Cancer 92(2) (2001), 276-284.

[26] J.P. Klussmann, S.J. Weissenborn, U. Wieland, V. Dries, J. Kolligs, M. Jungehuelsing et al., Prevalence, distribution, and viral load of human papillomavirus 16 DNA in tonsillar carcinomas, Cancer 92(11) (2001), 2875-2884.

[27] S.M. Dong, D.I. Sun, N.E. Benoit, I. Kuzmin, M.I. Lerman and D. Sidransky, Epigenetic inactivation of RASSF1A in head and neck cancer, Clin Cancer Res 9(10 Pt 1) (2003), 3635-3640.

[28] M. Gasco, A.K. Bell, V. Heath, A. Sullivan, P. Smith, L. Hiller et al., Epigenetic inactivation of 14-3-3 sigma in oral carcinoma: association with p16(INK4a) silencing and human papillomavirus negativity, Cancer Res 62(7) (2002), 20722076.

[29] B.J. Braakhuis, P.J. Snijders, W.J. Keune, C.J. Meijer, H.J. Ruijter-Schippers, C.R. Leemans et al., Genetic patterns in head and neck cancers that contain or lack transcriptionally active human papillomavirus, J Natl Cancer Inst 96(13) (2004), 998-1006.

[30] J. Mork, A.K. Lie, E. Glattre, G. Hallmans, E. Jellum, P. Koskela et al., Human papillomavirus infection as a risk factor for squamous-cell carcinoma of the head and neck, $N$ Engl $J$ Med 344(15) (2001), 1125-1131.

[31] Y. Sun, J. Eluf-Neto, N. Muñoz, M. Booth, J.M.M. Walboomers, K.V. Shah et al., Human papillomavirus-related serological marker of invasive cervical carcinoma in Brazil, Cancer Epidemiol Biomarkers Prev 3 (1994), 341-347.

[32] K. Zumbach, M. Hoffmann, T. Kahn, F. Bosch, S. Gottschlich, T. Gorogh et al., Antibodies against oncoproteins E6 and E7 of human papillomavirus types 16 and 18 in patients with head-and-neck squamous-cell carcinoma, Int J Cancer 18(6) (2000), 815-816.

[33] H. Mellin, S. Friesland, R. Lewensohn, T. Dalianis and E. Munck-Wikland, Human papillomavirus (HPV) DNA in tonsillar cancer: clinical correlates, risk of relapse, and survival, Int J Cancer 89(3) (2000), 300-304.

[34] K. Lindel, K.T. Beer, J. Laissue, R.H. Greiner and D.M. Aebersold, Human papillomavirus positive squamous cell carcinoma of the oropharynx: a radiosensitive subgroup of head and neck carcinoma, Cancer 92(4) (2001), 805-813.

[35] W. Li, C.H. Thompson, C.J. O'Brien, E.B. McNeil, R.A. Scolyer, Y.E. Cossart et al., Human papillomavirus positivity predicts favourable outcome for squamous carcinoma of the tonsil, Int J Cancer 106(4) (2003), 553-558.

[36] P.M. Weinberger, Z. Yu, B.G. Haffty, D. Kowalski, M. Harigopal, J. Brandsma et al., Molecular classification identifies a subset of human papillomavirus - associated oropharyngeal cancers with favorable prognosis, J Clin Oncol 24(5) (2006), 736-747.

[37] F. Coutlee, A.M. Trottier, G. Ghattas, R. Leduc, E. Toma, G. Sanche et al., Risk factors for oral human papillomavirus in adults infected and not infected with human immunodeficiency virus, Sex Transm Dis 24(1) (1997), 23-31.

[38] A.R. Kreimer, A.J. Alberg, R. Daniel, P.E. Gravitt, R. Viscidi, E.S. Garrett et al., Oral human papillomavirus infection in adults is associated with sexual behavior and HIV serostatus, J Infect Dis 189(4) (2004), 686-698.

[39] B.G. Hansson, K. Rosenquist, A. Antonsson, J. Wennerberg, E.B. Schildt, A. Bladstrom et al., Strong association between infection with human papillomavirus and oral and oropharyngeal squamous cell carcinoma: a population-based casecontrol study in southern Sweden, Acta Otolaryngol 125(12) (2005), 1337-1344.

[40] M. Frisch, R.J. Biggar and J.J. Goedert, Human papillomavirus-associated cancers in patients with human immunodeficiency virus infection and acquired immunodeficiency syndrome, J Natl Cancer Inst 92(18) (2000), 1500-1510.

[41] D.M. Parkin, The global health burden of infection-associated cancers in the year 2002, Int J Cancer 118(12) (2006), 30303044.

[42] M. Frisch, H. Hjalgrim, A.B. Jaeger and R.J. Biggar, Changing patterns of tonsillar squamous cell carcinoma in the United States, Cancer Causes Control 11(6) (2000), 489-495.

[43] L. Hammarstedt, D. Lindquist, H.M. Dahlstrand, M. Romanitan, L. Dahlgren, J. Joneberg et al., Human papillomavirus as 
a risk factor for the increase in incidence of tonsillar cancer, Int J Cancer 119(11) (2006), 2620-2623.

[44] K. Devaraj, M.L. Gillison and T.C. Wu, Development of HPV vaccines for HPV-associated head and neck squamous cell carcinoma, Crit Rev Oral Biol Med 14(5) (2003), 345-362.

[45] B.M. Steinberg, W.C. Topp, P.S. Schneider and A.L. Abramson, Laryngeal papillomavirus infection during clinical remission, $N$ Engl J Med 308 (1983), 1261-1264.

[46] B.J. Wiatrak, D.W. Wiatrak, T.R. Broker and L. Lewis, Recurrent respiratory papillomatosis: a longitudinal study comparing severity associated with human papilloma viral types 6 and 11 and other risk factors in a large pediatric population, Laryngoscope 114(11 Pt 2 Suppl 104) (2004), 1-23.

[47] P.M. Reidy, H.H. Dedo, R. Rabah, J.B. Field, R.H. Mathog, L. Gregoire et al., Integration of human papillomavirus type 11 in recurrent respiratory papilloma-associated cancer, Laryngoscope 114(11) (2004), 1906-1909.

[48] C.S. Derkay, Task force on recurrent respiratory papillomas: a preliminary report, Arch Otolaryngol Head Neck Surg 121 (1995), 1386-1391.

[49] T.A. Cook, A.M. Cohn, J.P. Brunschwig et al., Laryngeal papilloma: etiologic and therapeutic considerations, Ann Otol, Rhinol, Laryngol 82 (1973), 649.
[50] M.J. Silverberg, P. Thorsen, H. Lindeberg, L.A. Grant and K.V. Shah, Condyloma in pregnancy is strongly predictive of juvenile-onset recurrent respiratory papillomatosis, Obstet Gynecol 101(4) (2003), 645-652.

[51] K.V. Shah, E.R. Unger, C.S. Derkay and B.M. Steinberg, Recurrent respiratory papillomatosis: bright prospects for vaccine-based prevention, Papillomavirus Rep 16(6) (2005), 333-338.

[52] M.S. Strong, C.W. Vaughan and G.B. Healy, Recurrent respiratory papillomatosis, in: Laryngo-Tracheal Problems in the Pediatric Patient, M. Healy, ed., Springfield: Thomas, 1979, pp. $88-98$.

[53] L.L. Villa, R.L. Costa, C.A. Petta, R.P. Andrade, K.A. Ault, A.R. Giuliano et al., Prophylactic quadrivalent human papillomavirus (types 6,11,16, and 18) L1 virus-like particle vaccine in young women: a randomised double-blind placebocontrolled multicentre phase II efficacy trial, Lancet Oncol 6(5) (2005), 271-278.

[54] L.R. Armstrong, C.S. Derkay and W.C. Reeves, Initial results from the national registry for juvenile-onset recurrent respiratory papillomatosis. RRP Task Force, Arch Otolaryngol Head Neck Surg 125(7) (1999), 743-748. 


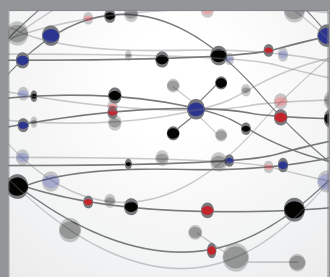

The Scientific World Journal
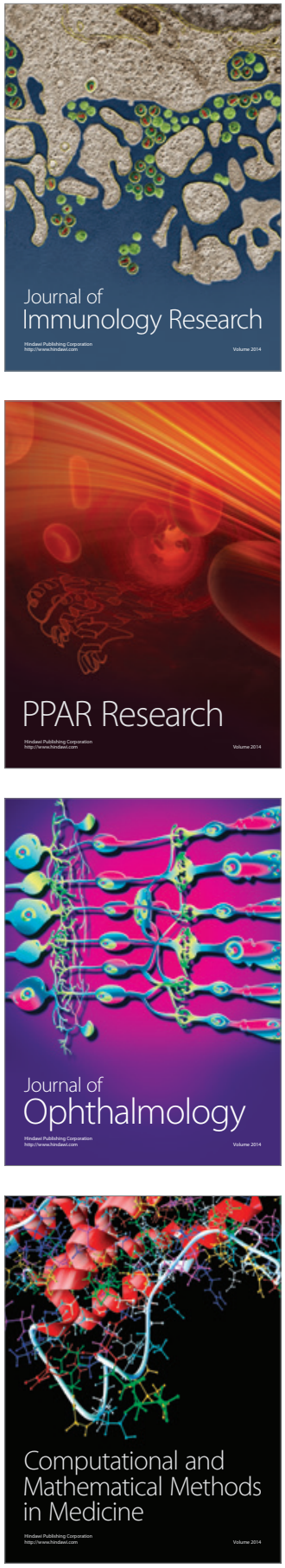

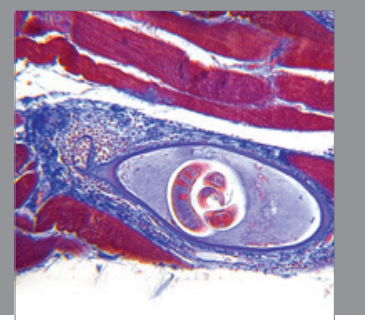

Gastroenterology

Research and Practice
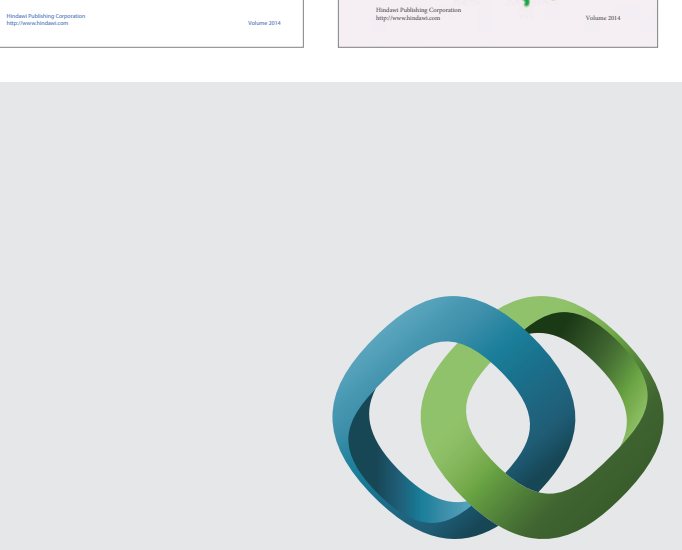

\section{Hindawi}

Submit your manuscripts at

http://www.hindawi.com
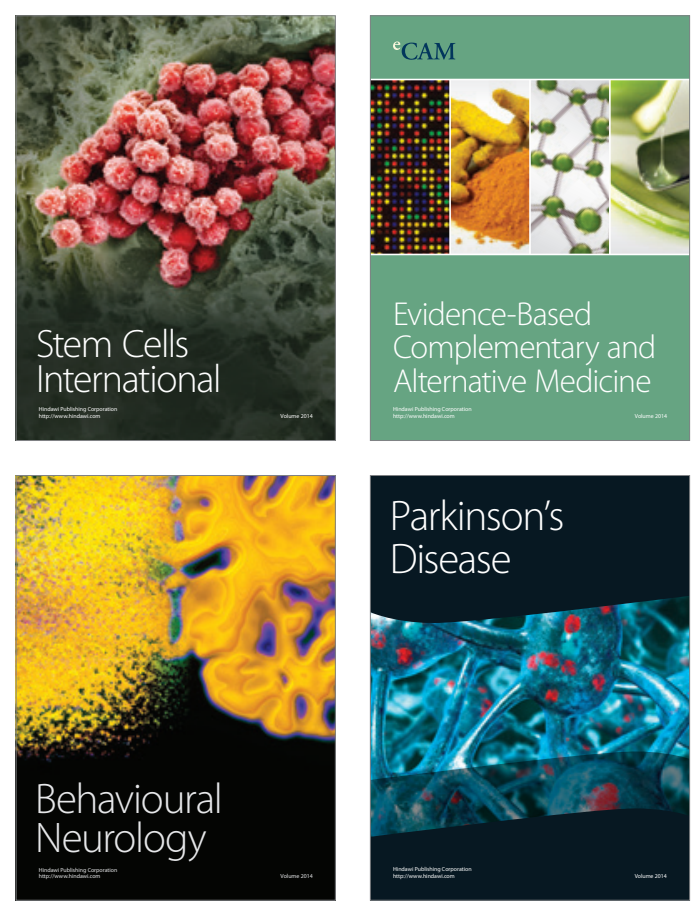

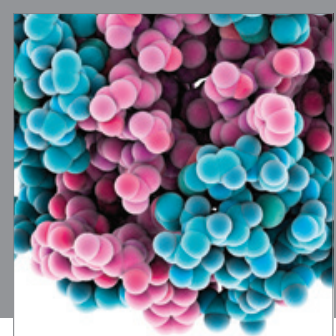

Journal of
Diabetes Research

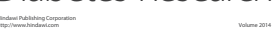

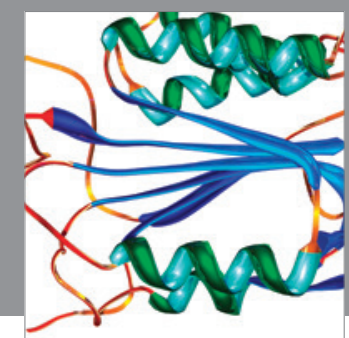

Disease Markers
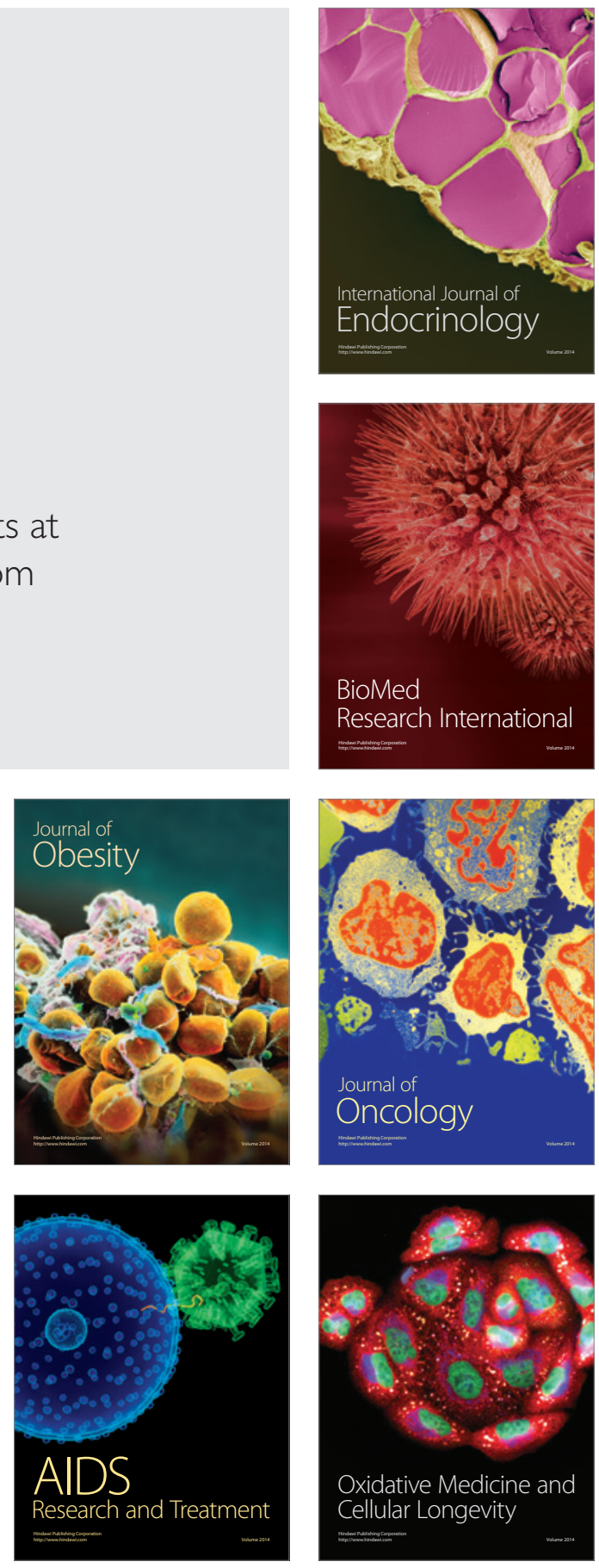